\begin{tabular}{ccc}
\hline & International Journal of Engineering \& Technology, $7(2.12)(2018) 90-92$ \\
SPC & International Journal of Engineering \& Technology \\
Website: $w w w . s c i e n c e p u b c o . c o m / i n d e x . p h p / I J E T$ & Research Paper \\
\hline
\end{tabular}

\title{
Development of iot furniture platform connected to smart home and application
}

\author{
Byung-Yoon Chun * \\ Dept. of Tax \& Management, 277Hyodeok-ro, Gwangju University, Nam-guGwangju city, 61743, KOREA \\ *Corresponding author E-mail: overcome@gwangju.ac.kr
}

\begin{abstract}
Background/Objectives: A practical use of sterilizer to prevent the inside of closet and drawer from going moldywill be expected to increase demand in sterilizer for earrings and accessories.

Methods/Statistical analysis: Application design and software are developed in consideration of user convenience connected with IoT platform. Major performance and function are defined, including materialization of unit function, hardware platform and instrument development, and development of embedded S/W and mobile application.

Findings: Application connected to closing and opening device based on IoT is developed not only to remotely monitor open and shut the door in closet or drawer but also to switch device remotely which controls ion generator and humidity but also to operate alarm device in case of opening door of closet or drawer forcibly.

Improvements/Applications: Application as a smart lock for security can be expected in office, closet, and drawers. A practical use of sterilizer to prevent the inside of closet and drawer from going moldy is expected to using as a sterilizer for earrings and accessories.
\end{abstract}

Keywords: Smart Home; IO; Embedded; Closing and Opening Device; Smart Furniture; PCB.

\section{Introduction}

Device for closing and opening smart furniture based on IoT and interlocking application are developed to open and close door in closet, drawer, and dressing table through fingerprint verification, including promoting a pleasant environment to prevent the inside of closet and drawer from going moldy. Product design not only to open and close door remotely by smartphone but to control and monitor various kind of devices based on IoT is also to be developed in this study [1], [2]. Therefore, the development of following contents is to be implemented; the product design considered the body in die production, mass production structure for securing proper manufacturing costs, the development of application connected device of closing and opening based on IoT, the design of control unit and fingerprint recognition part, the development of detailed circuit diagram and PCB artwork and the development of control firmware [3], [4].

\section{Research and development}

\subsection{Development of IoT platform connected to smart home}

Device of opening and closing closet door through fingerprint identification is developed under $12 \mathrm{~V}$ power adapter located the back side of closet or drawer. Manufacturing extra board not only to store source image of finger print DB in control board memory but to install fingerprint identification sensor in the outside of drawer or closet is to be connected main control board by F-PCB [5], [6]. A number of anions which are created by air electrolysis purifies the air and have an effect on odor removing, suppression of propagation of germs, and resolving harmful gas. Humidity measurement inside closet using humidity sensor and humidity control device using electric motor driven fan are also developed. Operation condition of opening and closing door as well as fan is transmitted and is managed smart phone through WiFi gate [7], [8].

Control unit block diagram of furniture based on IoT device is shown in figure 1 .

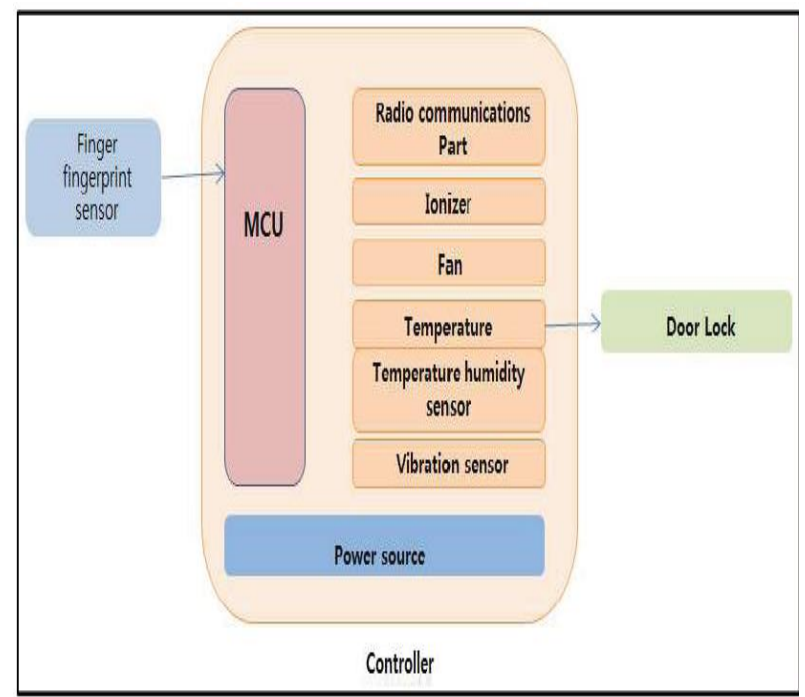

Fig. 1: Control Unit Block Diagram in Furniture Based on Iot Device.

Wire frame for the development of application connected to closing and opening device based IoT is shown in Figure 2. 


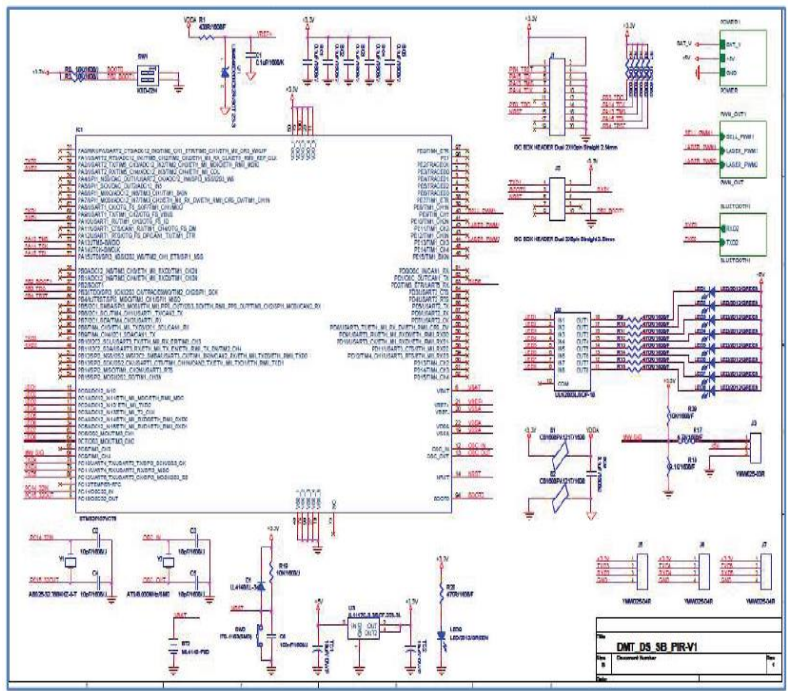

Fig. 2: Picture of Circuit Design.

The mobile application result of furniture based IoT is shown in Figure 3. Its major functions are as in the flowing; remotely closing and opening door of closet or drawer, remotely monitoring door condition, remotely switching an ion generator and a humidity control device, and alarm operating in case of opening door forcibly9, 10 .

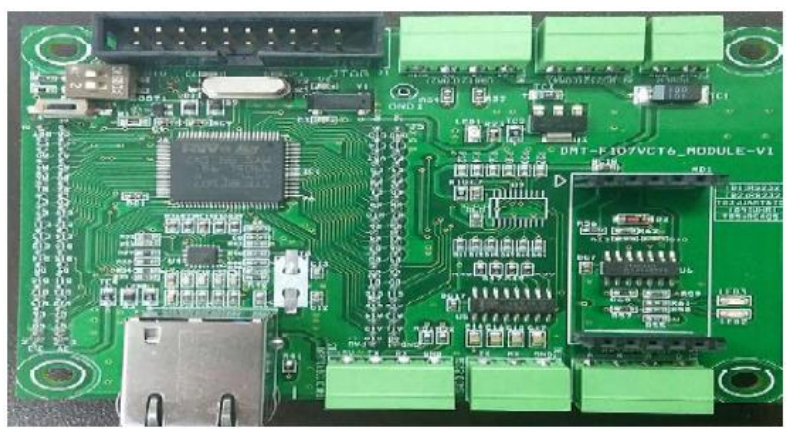

Fig. 3: Picture of PCB Manufacturing

Main controller artwork is shown in figure 4.

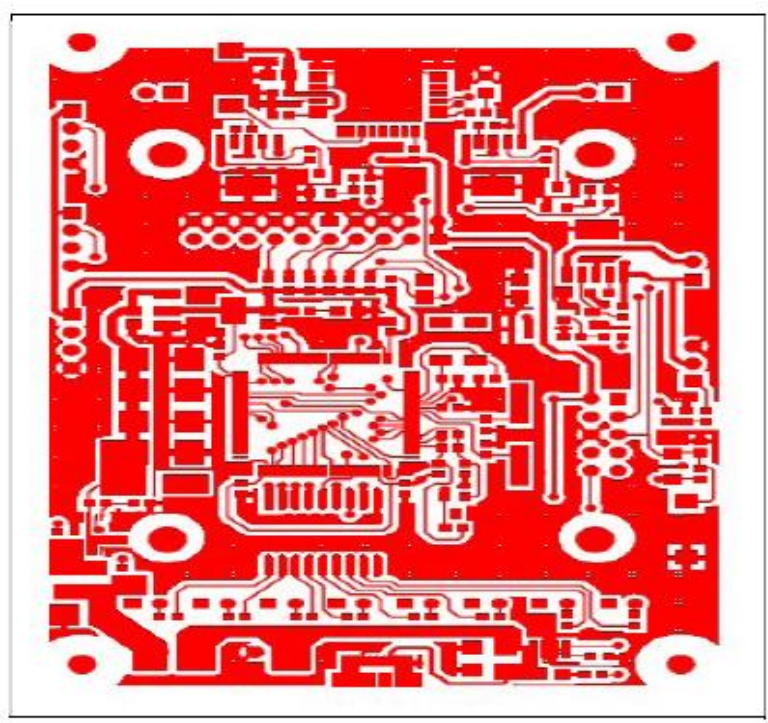

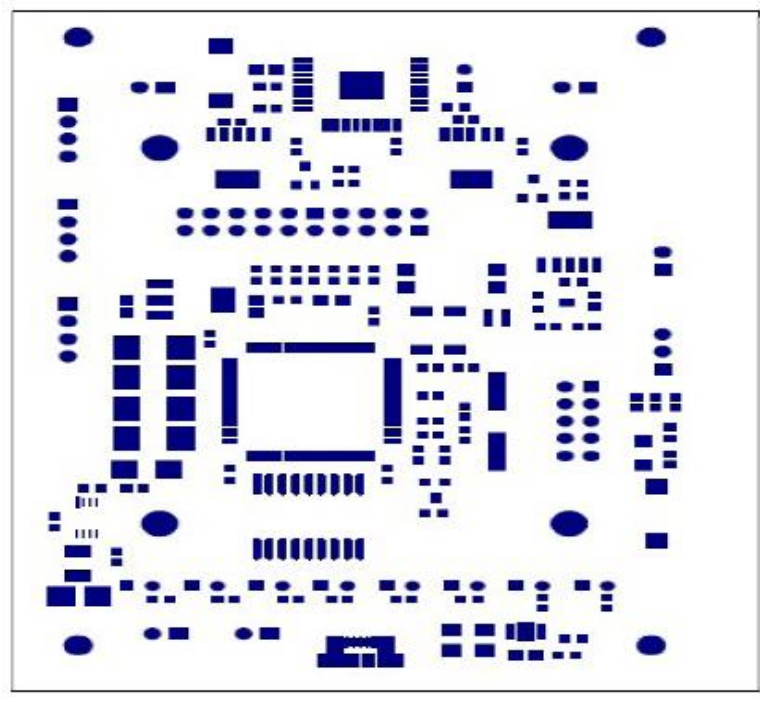

Fig. 4: Artwork of Main Controller.

Picture of final PCB manufacturing completion is shown in figure in 5
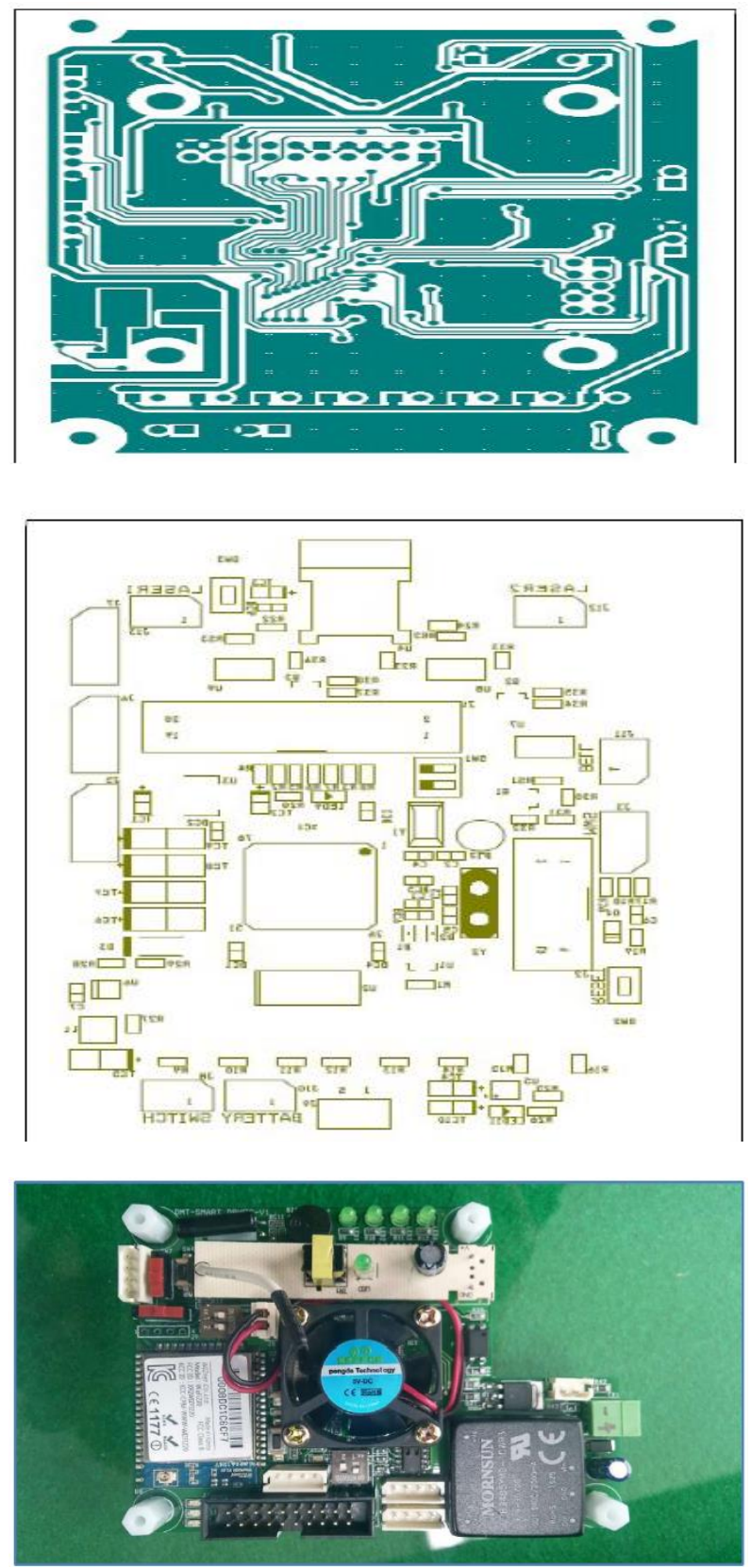

Fig. 5: Picture of Final PCB Product. 


\subsection{Development of product design}

Products such as electrical wirings of every kind, cooling fan, sensor and battery are designed. Product design which is considered production of body mold and exploration into utilization method of additional function as well as design type are developed. Product design is shown in figure 6 .

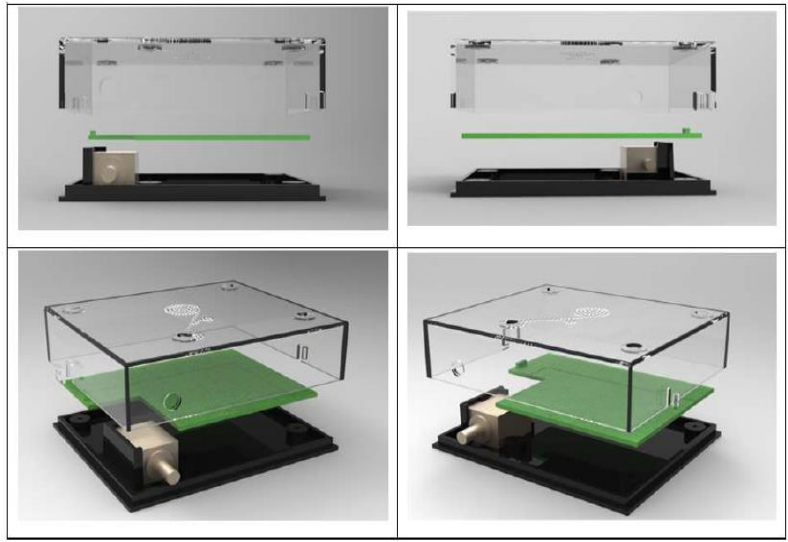

Fig. 6: Product Design.

\subsection{Development of application connected to closing nd} opening device based on IoT.

Android-Nougat platform has been developed. Wire frame for development of application connected to closing and opening device based on IoT is shown in figure 7.

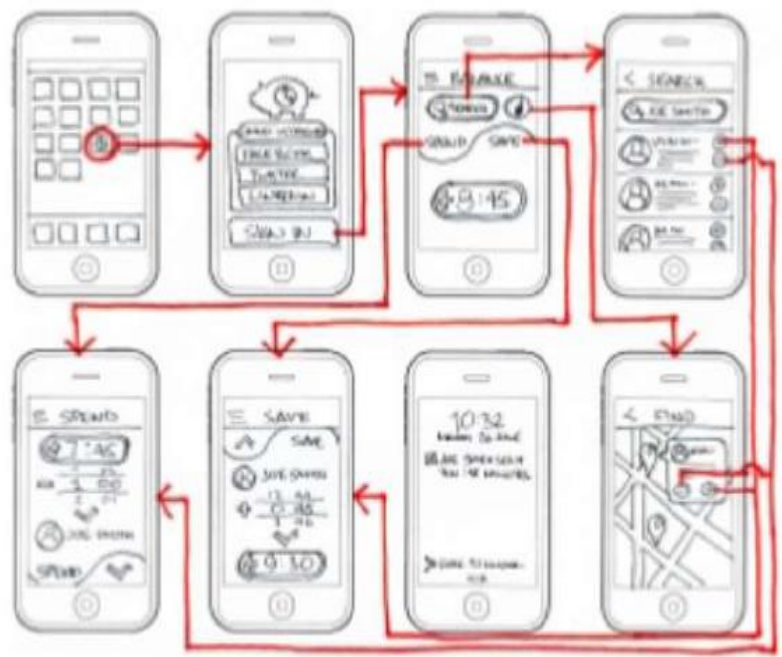

Fig. 7: Wire Frame of Mobile Application in Furniture Based on IoT.

Developing result of mobile application in furniture based on IoT is shown in figure 8.
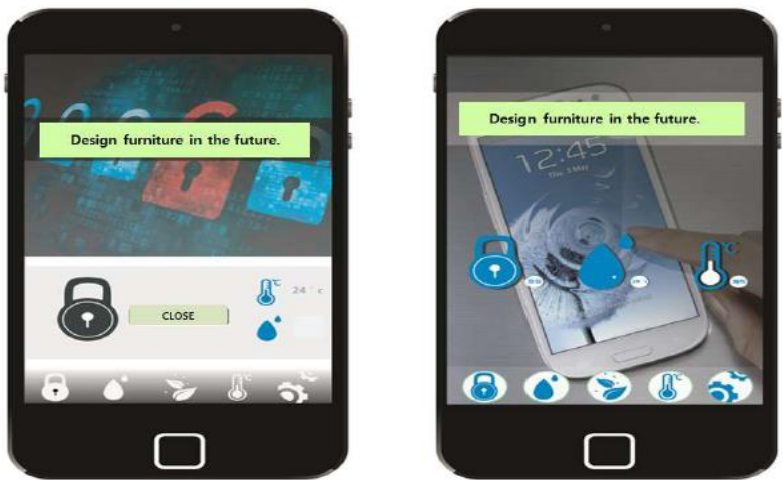

Fig. 8: Developing Result of Mobile Application in Furniture Based on IoT.

\section{Conclusion}

Application connected to closing and opening device based on IoT is developed not only to remotely monitor open and shut the door in closet or drawer but also to switch device remotely which controls ion generator and humidity but also to operate alarm device in case of opening door of closet or drawer forcibly. Market expansion of application as well as smart home through the development of furniture IoT device connected to smartphone based on this study is trying to contribute to develop local industries. Removing the mold from drawers in home and office is applicable to a sterilizing device that is possible to prevent going moldy on clothes and accessories such as earring and necklace. It will be expected to use remote monitoring and controlling furniture based IoT.

\section{Acknowledgment}

This Study was conducted by research funds from Gwangju University in 2018 , Korea

\section{References}

[1] Bleda, A. L., Fernandez-Luque, F. J. , Rosa, A. , Zapata, J., Maestre, R., Smart Sensory Furniture Based on WSN for Ambient Assisted Living, IEEE SENSORS JOURNAL. 2017, 17(17), pp. 5626-5636.

[2] Tang, J.T. , He, H.Y. , Wu, X., Design and Implementation of the System Based on the Mechanical Topology Smart Furniture, Applied Mechanics and Materials. 2013, 329(-), pp. 497.

[3] Hu, Xiao-Jun , Xu, Feng, Li, Qian, A Transition from Microstrip to SINRD Waveguide of PCB Version, ACTA ELECTRONICA SINICA. 2017, 45(8), pp. 1985-1988.

[4] Atago, Yuki, Shimodaira, Jun, Araki, Naoto, Bin Othman, Nor'azizi , Zakaria, Zuriati , Fukuda, Masao , Futami, Junichiro ,Hara, Hirofumi, Identification of novel extracellular protein for $\mathrm{PCB} / \mathrm{bi}$ phenyl metabolism in Rhodococcusjostii RHA1, Bioscience, Biotechnology, and Biochemistry. 2016, 80(5), pp. 1012-1019.

[5] Sensinger, Jon W. , Lipsey, James , Thomas, Ashley, Turner, Kristi, Design and evaluation of voluntary opening and voluntary closing prosthetic terminal device, JOURNAL OF REHABILITATION RESEARCH AND DEVELOPMENT. 2015, 52(1), pp. 63-76.

[6] Enpuku, K. , Tokimizu, D. , Kuroda, D. , Hijiya, S., A Four-Junction Switch for Controlling the Opening and Closing of a Pickup Coil in High-T c Superconducting Quantum Interference Device Magnetometer, Japanese Journal of Applied Physics. 2001, 40(88), pp. L869-L871.

[7] Hildebrandt, Julian , Brauner, Philipp , Ziefle, Martina, Smart Textiles as Intuitive and Ubiquitous User Interfaces for Smart Homes, Lecture Notes in Computer Science. 2015, 2015(9194), pp. 423-434.

[8] Anvari-Moghaddam, A. , Monsef, H. , Rahimi-Kian, A., Optimal Smart Home Energy Management Considering Energy Saving and a Comfortable Lifestyle, IEEE Transactions on Smart Grid. 2015, 6(1), 324-332.

[9] Marri, S., Ratha, S. , Rout, C., Behera, J. N., 3D cuboidal vanadium diselenide embedded reduced graphene oxide hybrid structures with enhanced supercapacitor properties, Chemical communications. 2017, 5391), pp. 228-231.

[10] Oh, S. , Lee, K. , Kim, S. J. , Ha, K. , Jeong, J. , Kim, D. , Kim, K. K. , Park, S. J., Self-assembled indium tin oxide nanoball-embedded omnidirectional reflectors for high photon extraction efficiency in III-nitride ultraviolet emitters, Nanoscale. 2017, 9(22), pp. 7625 3372 . 